\title{
GERMANIUM BAND GAP ENGINEERING INDUCED BY TENSILE STRAIN FOR SI-BASED OPTOELECTRONIC APPLICATIONS
}

\author{
LUONG THI KIM PHUONG \\ Aix Marseille University, CNRS, CINaM-UMR 7325, F-13288 Marseille, France \\ and \\ Hong Duc University, 565 Quang Trung St., Thanh Hoa City, Vietnam \\ NGUYEN MANH AN \\ Hong Duc University, 565 Quang Trung St., Thanh Hoa City, Vietnam
}

Received 19 August 2013

Accepted for publication 24 December 2013

\begin{abstract}
We have combined structural and optical characterizations to investigate the tensilestrained state and the band gap engineering of Ge layers grown on Si(001) using molecular beam epitaxy. The tensile strain is generated in the Ge layers due to a difference of thermal expansion coefficients between Ge and Si. The Ge growth on Si(001) was proceeded using a two-step growth process: a low-temperature step to produce relaxed buffer layers, followed by a high-temperature step to generate the tensile strain in the Ge layers. For the low-temperature step, we have evidenced the existence of a substrate temperature window from 260 to $300^{\circ} \mathrm{C}$ in which the well-known StranskiKrastanov Ge/Si growth mode transition from two-dimensional to three-dimensional growth can be completely suppressed. We show that the value of the tensile strain in the Ge layers lineally increases with increasing the growth temperature and reaches a saturation value of $\sim 0.24 \%$ in the temperature range of $700-770^{\circ} \mathrm{C}$. Post-grown cyclic thermal annealing has allowed to increase the tensile strain up to $0.30 \%$, which is the highest value ever reported to date. Finally, photoluminescence measurements reveal both an enhancement of the Ge direct band gap emission and a reduction of its energy due to the presence of tensile strain in the layers.
\end{abstract}

\section{INTRODUCTION}

Silicon $(\mathrm{Si})$, germanium $(\mathrm{Ge})$ and their alloys are the main materials that are used as active layers in microelectronics. However, due to their indirect band gap the realization of optoelectronic devices, in particular light emitting devices, has been essentially based on III-V or II-VI semiconductors. Recently, it has been shown that strong optical gain can be obtained in tensile-strained and $n$-doped Ge layers and laser emission has been demonstrated under optical pumping using Ge as active layers [1]. Compared to Si or SiGe alloys, pure Ge displays unique optical properties. Indeed, the direct $(\Gamma)$ valley of its conduction band is only $0.14 \mathrm{eV}$ above the indirect $(L)$ valley at room temperature while it is larger than $2 \mathrm{eV}$ in $\mathrm{Si}$. It has been shown that when a tensile strain is applied to Ge, the energy position of the conduction $\Gamma$ valley decreases faster than the one of the band-edge $L$ valley [2,3] and a transition from indirect to direct band gap can occur for an 
in-plane tensile strain of $\sim 1.9 \%$ [4]. On the other hand, $n$-type doping of Ge leads to a more efficient population of the zone center $\Gamma$ valley and thus enhances optical recombination at the Brillouin zone center $[5,6]$.

Tensile strain can be induced in Ge via several approaches: applying external mechanical stress [7], growing Ge on a larger lattice parameter substrate, such as InGaAs [8] or GeSn buffer layers [9], or by taking benefit of the thermal mismatch between Ge and $\mathrm{Si}[1,5]$.Among these approaches, the latter is of particular interest since it allows direct integration of Ge-based optoelectronic devices into the mainstream Si technology. However, due to the existence of a misfit as high as $4.2 \%$ between $\mathrm{Ge}$ and $\mathrm{Si}$, it is crucial to control both the crystalline quality and the surface roughness of the Ge epilayers. Indeed, the growth of Ge on $\mathrm{Si}$ is considered as a typical example of the Stranski-Krastanov (SK) mode: a two-dimensional (2D) wetting layer is formed only for film thicknesses below a critical thickness of some atomic monolayers (ML), beyond which a transition to a three-dimensional (3D) islanding growth mode occurs to relieve the built-in strain in the Ge layers $[10,11]$. As a result of the plastic strain relaxation, Ge films with larger thicknesses display a high density of threading dislocations and a rough surface. To prevent 3D nucleation of Ge islands, a two-step growth technique has been proposed, which consists of a low-temperature growth to form a strain-relaxed layer, followed by a growth at higher temperatures to produce thicker films [12,13]. This growth techniquehas proven its efficiency regarding the reduction of the density of threading dislocations and also the surface roughness $[1,5,12-15]$. However,it is worth noting that in all above-mentioned experiences, the chemical-vapor deposition (CVD) technique has been exclusively used. While the low-temperature growth step is recognized to be crucial to control the final state of the film crystalline quality, the degree of freedom regarding the choice of the substrate temperature remains limited within the CVD technique. Indeed, in a standard CVD process, the thermal energy provided by the substrate temperature should be high enough to dissociate the gas precursor molecules. For this reason, in previous works the low-temperature step has been indeed carried out at intermediate temperatures ranging between 350 and $400^{\circ} \mathrm{C}[1,5,12-15]$. In addition, the presence of hydrogen on the growing surface that comes from hydrogen carrier gas or decomposition of hydride molecules may reduce the surface diffusion length of Ge adatoms [16]. For example, it has been shown that Ge films grown by CVD at a temperature reduced down to $330^{\circ} \mathrm{C}$ present a high density of stacking faults and defects [17].

In this work, we report results of controlling tensile strain in Ge epilayers grown on $\mathrm{Si}(001)$ substrates using molecular-beam epitaxial (MBE) growth technique. One of the advantages of the MBE technique is that it does not need high growth temperatures to dissociate gas precursors. Ge films were grown using a two-step procedure. Concerning the growth of the first Ge layer, our purpose aims to determine growth conditions that allow getting strain-relaxed and smooth Ge layers. We have then investigated, in the first set of experiments, the Ge growth mode as a function of the substrate temperature within a wide temperature range, going from room temperature up to $550^{\circ} \mathrm{C}$. We have evidenced the existence of a narrow substrate temperature window, from 260 to $300^{\circ} \mathrm{C}$, in which the Stranski-Krastanov growth of Ge on Si can be completely suppressed. Instead, a two-dimensional growth mode is obtained up to film thicknesses larger than $300 \mathrm{~nm}$. In 
the second set of experiments, we have investigated the effect of the growth temperature and annealing condition on the strain level of Ge epilayers and obtained a strain value of $0.30 \%$, which represents the highest value ever reported.

\section{EXPERIMENTAL SET-UP}

Ge growth was performed in a standard solid source molecular beam epitaxial (MBE) system with a base pressure better than $5 \times 10^{-10}$ mbar. The growth chamber is equipped with a $30-\mathrm{keV}$ reflection high-energy electron diffraction (RHEED) apparatus allowing monitoring in real time the Ge growth mode. An Auger electron spectrometer (AES) is used to control the cleanliness of the substrate surface prior to growth and the film composition. Ge was evaporated from a two-zone heated Knudsen effusion cell to avoid Ge condensation at the upper part of the cell crucible, thus insuring a highly stable Ge deposition rate. The Ge deposition rate, measured using RHEED intensity oscillations during Ge homoepitaxy on a $\mathrm{Ge}(111)$ substrate, was in the range from 1.5 to $5 \mathrm{~nm} / \mathrm{min}$.

The substrates were flat, $p$-type $\mathrm{Si}(001)$ wafers. The cleaning of the Si surfaces was carried out using the HF-last dip method to obtain a hydrogen passivated Si surface [18]. In-situ thermal annealingwas performed at $\sim 650^{\circ} \mathrm{C}$ to remove the hydrogen passivated layer. After this step, the Si surface exhibits a well-developed $2 \times 1$ reconstruction and AES measurements do not reveal any presence of oxygen or carbon. The substrate temperature was measured using a thermocouple in contact with the backside of Si wafers. These measurements were corrected using an infrared pyrometer (Ircon, W-series) operating in the wavelength region of $0.90-1.08 \mu \mathrm{m}$, in which the emissivity of $\mathrm{Si}$ is constant. The accuracy of the temperature measurement is estimated to be about $\pm 20^{\circ} \mathrm{C}$.

Structural analysis of post-grown films was performed by means of high-resolution transmission electron microscopy (TEM) using a JEOL 3010 microscope operating at $300 \mathrm{kV}$ with a spatial resolution of $1.7 \AA$. The film surface morphology was measured using an Omicron atomic-force microscopy (AFM) in contact mode. The strain level in the Ge epilayers was deduced from X-ray diffraction (XRD) measurements performed using a diffractometer (Philips X'pert MPD) equipped with a copper target for $\mathrm{Cu}-\mathrm{K}_{\alpha 1}$ radiation $(\lambda=1.54059 \AA)$. The angular resolution is $\sim 0.01^{\circ}$. For the photoluminescence (PL) experiments, the samples were excited by a $532 \mathrm{~nm}$ laser (total power $13 \mathrm{~mW}$ ) focused on the sample surface through a $\mathrm{x} 40$ objective. The emitted light was then collected and analyzed by a monochromator equipped with a 600 grooves/mm grating and by a liquid nitrogen cooled InGaAs detector. All PL measurements were performed at room temperature.

\section{RESULTS AND DISCUSSION}

As compared to material growth in a CVD reactor, the MBE growth chamber is equipped with RHEED, which is a powerful technique allowing us to monitor in real-time the change of both the surface morphology and the surface structure of the growing film. By means of RHEED, we have investigated the Ge growth in a large temperature range, from room temperature up to $550^{\circ} \mathrm{C}$, and have evidenced the existence of a narrow window of the substrate temperature ranging from 260 to $300^{\circ} \mathrm{C}$ in which the Stranski-Krastanov 
(SK) Ge/Si growth can be completely suppressed. In this temperature window, twodimensional (2D) RHEED patterns of the Ge growing surface are continuously observed from the first layer up to a film thickness of a few hundreds of nm. Figure 1(a) shows a RHEED pattern taken along the [100] azimuth of a 300 nm thick Ge layer grown on $\mathrm{Si}(001)$ at a substrate temperature of $300^{\circ} \mathrm{C}$. The observation of long streaks in this RHEED pattern clearly indicates that the Ge film grows in a layer-by-layer (2D) mode and the formation of three-dimensional (3D) islands has been completely suppressed.The measurement of the in-plane lattice parameters from RHEED confirms that the Ge layers are almost fully relaxed. Figure 1(b) displays an AFM image of the corresponding surface. The surface appears highly smooth, no presence of $3 \mathrm{D}$ islands is visible. The average surface roughness is about $0.3 \mathrm{~nm}$, which is in good agreement with the observation of a 2D RHEED pattern, shown in Fig. 1(a). We can then conclude that we have succeeded to form relaxed and smooth Ge buffer layers, which serve as a support for the growth of tensile-strained Ge films at higher growth temperatures. It is also worth noting that to our knowledge Ge/Si films grown by CVD cannot produce such a high quality of the film surface.
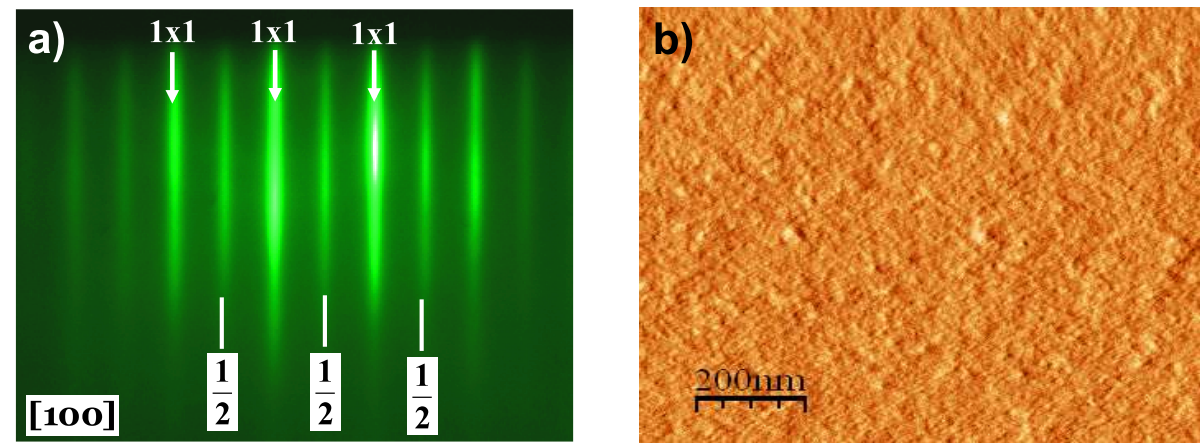

Fig. 1. (a): RHEED pattern along the [100] azimuth observed at the end of a $\sim 300 \mathrm{~nm}$ thick Ge layer grown at $300^{\circ} \mathrm{C}$. The observation of long streaks in the RHEED pattern clearly indicates that the formation of 3D islands has been completely suppressed. Note that $(1 \mathrm{x} 1)$ corresponds to the position of the Ge bulk-like streaks and $1 / 2$ is half-order streaks of the $2 \times 1$ reconstruction of the Ge(001) surface; (b) AFM image measured at room temperature using contact mode of the corresponding sample.

Afterthe low-temperature growth step, we have studied the effect of the hightemperature growth on the value of the tensile strain generated in the Ge layers. All samples investigated here have a total thickness of $\sim 300 \mathrm{~nm}$, of which $50 \mathrm{~nm}$ were first deposited at $300^{\circ} \mathrm{C}$ followed by a $250 \mathrm{~nm}$ thick Ge film deposited at various temperatures: $400,500,600,650,700,750$ and $770^{\circ} \mathrm{C}$. Figure 2 displays some representative $\theta-2 \theta$ XRD scans taken around the $\mathrm{Ge}(004)$ reflection. For comparison, we report in dotted lines a $\mathrm{XRD}$ scan of a sample grown at $300^{\circ} \mathrm{C}$. The fact that the (004) peak of this sample is located at $2 \theta \sim 66^{\circ}$, a value close to that measured on a Ge substrate, indicates that the corresponding Ge layer is almost fully relaxed. As can be seen in Fig.2(a), the $2 \theta$ angle 
value of the (004) reflection is found to linearly increase when the growth temperature increases from 300 to $700^{\circ} \mathrm{C}$ then remains almost constant for further increasing the temperature up to $770{ }^{\circ} \mathrm{C}$. We note that to obtain the value of the in-plane tensile strain $\varepsilon_{/ /}$, we first determine the out-of-plane strain $\varepsilon_{\perp}$ from the $\theta-2 \theta$ XRD curves and then deduce the value of $\varepsilon_{/ /}$using the following relationship: $\varepsilon_{/ /}\left(\varepsilon_{/ / /}+\varepsilon_{\perp}\right)=\mathrm{c}_{11} /\left(\mathrm{c}_{11}+2 \mathrm{c}_{12}\right)$ with $c_{11}=12.85 \times 10^{10} \mathrm{~Pa}$ and $\mathrm{c}_{12}=4.83 \times 10^{10} \mathrm{~Pa}$ for pure Ge [19]. The highest value of the in-plane tensile strain $\varepsilon_{/ /}$obtained in the growth temperature range of $700-770^{\circ} \mathrm{C}$ is $0.24 \%$, which is in good agreement with previous results reported using CVD in which the highest tensile strain was in the range of $0.22-0.25 \%[1,13-15,20]$ at similar growth temperatures. Shown in Fig. 2(b) is a summary of the evolution of the in-plane tensile strain versus the growth temperature. It is worth noting that for a Ge growth at a temperature higher than $800^{\circ} \mathrm{C}$, long-range $\mathrm{Ge} / \mathrm{Si}$ inter diffusion has been found to occur and the above elastic constants of pure Ge cannot be used to determine the value of the strain in the grown layers.

After growth, the samples were subjected to thermal anneals to reduce the density of threading dislocations but also to increase the tensile strain. Indeed, once the Ge epilayer has a certain tensile strain, upon annealing it will not behave as a perfect elastic material in which the stress-strain characteristic is ideally linear and the strain variation curve is perfectly superimposed over the stress variation curve. The Ge epilayer will behave visco-elastically and nonlinear behavior of stress-strain curve appears. Two methods of thermal annealing have been used, a long anneal at $900^{\circ} \mathrm{C}$ and cyclic anneals from 780 to $900^{\circ} \mathrm{C}$. It has been found that cyclic annealingis more efficient regarding the introduction of additional strain in the Ge films. The XRD curve measured after ten annealing cycles from 780 to $900^{\circ} \mathrm{C}$ with an annealing time of $3 \mathrm{~min}$ at each temperature is shown in Fig. $2\left(\varepsilon=0.30 \%\right.$ solid curve). The as-grown sample was deposited at $600^{\circ} \mathrm{C}$ and its corresponding in-plane tensile strain is $0.16 \%$. As can be seen, after cyclic annealing, the tensile strain in the layer has increased up to $0.30 \%$. The above result indicates that an adequate combination of the two-step growth method, in particular the control of the low-temperature growth step, with cyclic annealing allows to get Ge epilayers having a high crystalline quality, a smooth surface and also a high level of tensile strain.

It is worth noting that the tensile-strain determination from asymmetric (224) and $(-2-24)$ is in good agreement with the above values deduced from the symmetric (004) reflection. In addition, we have also proceeded cyclic annealing for samples grown at 650 and $700^{\circ} \mathrm{C}$ and a similar value of tensile strain was obtained. These results clearly confirm the effectiveness of the cyclic annealing to a further increase the tensile strain in the Ge epilayers. However, we have found out that the increase of the tensile strain and $\mathrm{Ge} / \mathrm{Si}$ interdiffusion are competing processes, so that for a sample that has been grown at a high temperature, for example at $770^{\circ} \mathrm{C}$, the number of annealing cycles and the annealing time should be optimized to control the degree of $\mathrm{Ge} / \mathrm{Si}$ interdiffusion.

Figure 3 shows an overall TEM image of a sample grown at $730^{\circ} \mathrm{C}$. The image clearly indicates thatthe Ge epilayer has a high crystalline quality and a relatively uniform thickness. Of particular interest, stacking faults aligned along (111) planes, which are commonly observed in strain-relaxed $\mathrm{Ge} / \mathrm{Si}$ epilayers grown at temperatures higher than $\geq 330^{\circ} \mathrm{C}$ are almost absent (see, for example, Fig. 10 of ref [17] using CVD). Furthermore, 


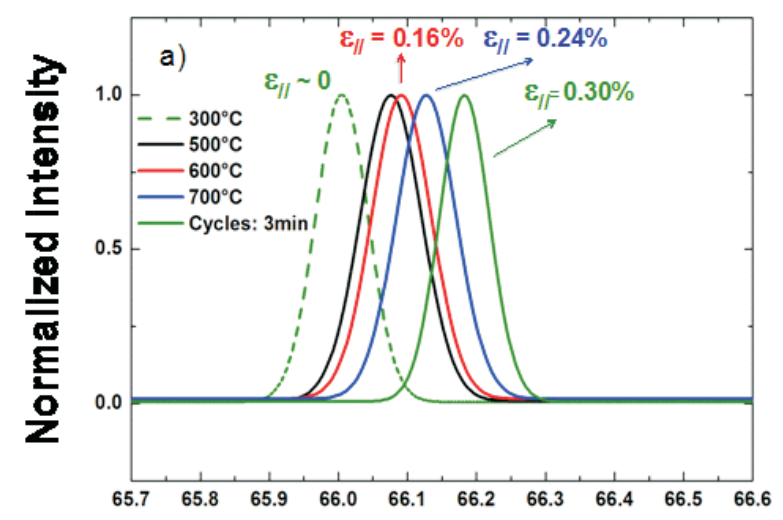

$\mathbf{2 0}\left({ }^{\circ}\right)$

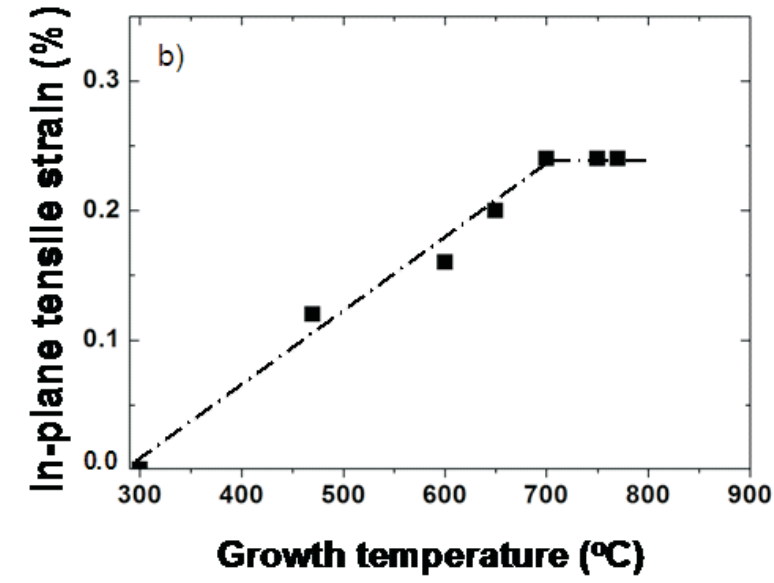

Fig. 2. Evolution of $\theta-2 \theta$ XRD scans around the Ge(004) reflection versus the growth temperature and post-growth thermal annealing. The dotted XRD scan, which corresponds to a sample grown at $300{ }^{\circ} \mathrm{C}$, is shown for comparison. As the growth temperature increases, the $\mathrm{Ge}(004)$ reflection linearly shifts to higher angles, reaching a saturation value at $700^{\circ} \mathrm{C}$, and finally remains almost constant for further increasing the temperature to $770^{\circ} \mathrm{C}$. The in-plane tensile strain observed in the temperature range $700-770^{\circ} \mathrm{C}$ is $0.24 \%$. The curve on the right $\left(\varepsilon_{/ /}=0.30 \%\right.$ solid curve) corresponds to 10 annealing cycles from 780 to $900{ }^{\circ} \mathrm{C}$ with an annealing time of 3 min at each temperature. After annealing, a tensile strain as high as $0.30 \%$ has been reached; (b) Summary of the evolution of the in-plane tensile strain versus the growth temperature.

the film surface appears relatively smooth, which is well correlated with the observation of streaky RHEED patterns during Ge deposition at this temperature. The image also reveals the presence of a high density of misfit dislocations, which have allowed strain relaxation to take place in the Ge epilayer. The most interesting feature observed here is that unlike the case of Ge films grown by CVD [19], here we observe that most of misfit 
dislocations are located close to the interface and only a few of them have penetrated into the Ge epilayer. This explains why the observed interface is relatively smooth and well defined.

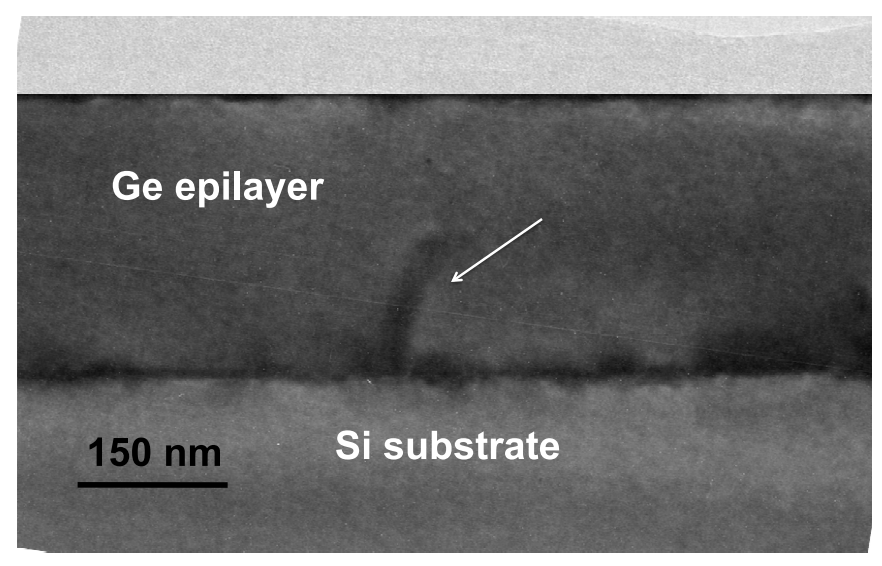

Fig. 3. Typical cross-sectional TEM image of a $\sim 300 \mathrm{~nm}$ thick Ge layer grown following a two-step growth at $300 / 730^{\circ} \mathrm{C}$. A threading dislocation, indicated by white arrows, appears in the layer during the increase to the high-temperature growth step. However, compared to Ge films grown using CVD, the present layer exhibits an extremely low density of threading dislocations.

Figure 4 shows the evolution of the photoluminescence (PL) spectrum of Ge films with the growth temperature. All Ge films have the same thickness and it is worth noting that PL measurements were carried out at room temperature. For a film grown a $300^{\circ} \mathrm{C}$, the PL spectrum peaks at around $1525 \mathrm{~nm}$, which can be attributed to arise from the direct band gap radiative recombination of relaxed Ge. Note that the wavelength corresponding to the indirect band gap of Ge should be located above $1800 \mathrm{~nm}$, which is out of the spectral range due to detector cut-off. With increasing the growth temperature to 600$650{ }^{\circ} \mathrm{C}$, the photoluminescence is found to slightly shift to longer wavelengths. A redshift of $\sim 19 \mathrm{~nm}$ is observed at $650^{\circ} \mathrm{C}$ as compared to relaxed Ge, which can be attributed to the reduction of the direct band gap due to the presence of tensile strain in the Ge film. When the growth temperature increases to $700^{\circ} \mathrm{C}\left(\varepsilon_{/ /} \sim 0.24 \%\right)$, we do not observe further redshift, the corresponding PL spectrum is almost superimposed on the $650^{\circ} \mathrm{C}$ spectrum. A feature of interest is that with further increasing the growth temperature to $750^{\circ} \mathrm{C}$, the $\mathrm{PL}$ is found to quench $\left(\varepsilon_{/ /}=0.30 \%\right.$ curve). Such a quenching of PL at $750^{\circ} \mathrm{C}$ can be attributed to $\mathrm{Ge} / \mathrm{Si}$ interdiffusion even if XRD and SIMS measurements (not shown here) do not reveal pronounced interdiffusion at this temperature. However, one should keep in mind that XRD and SIMS are macroscopic characterization tools while probing the optical properties of Ge should be much more sensitive to nanoscaled $\mathrm{Ge} / \mathrm{Si}$ interdiffusion and the presence of a very small amount of $\mathrm{Si}$ in Ge layers can greatly reduce the radiative recombination in the layer because the band structure between Si and Geat the Brillouin zone center is highly difference. 


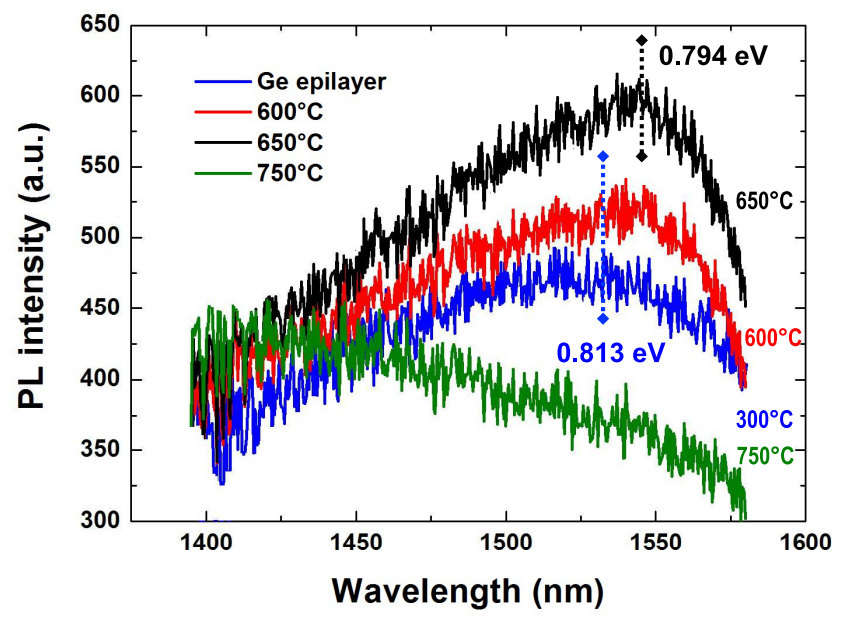

Fig. 4. Evolution of the PL spectrum measured at room temperature of Ge films versus the growth temperature. The photoluminescence arising from Ge direct band gap is shown to slightly shift to longer wavelengths with increasing the growth temperature from 300 to $650^{\circ} \mathrm{C}$. At $750^{\circ} \mathrm{C}$, the PL is quenched, which can be attributed to $\mathrm{Ge} / \mathrm{Si}$ interdiffusion.

\section{CONCLUSION}

We have investigated the growth of tensile-strained Ge films on Si substrates using a two-step growth method by molecular beam epitaxy. We have found that the first growth step is crucial to determine not only the film crystalline quality, the surface morphology but also the final strain state in the Ge films. We have evidenced the existence of a narrow window of the substrate temperature from 260 to $300^{\circ} \mathrm{C}$ in which it the $\mathrm{Ge} / \mathrm{Si}$ Stranski-Krastanov growth can be completely suppressed. We have investigated the effect of both the growth temperature and annealing parameter to the strain state in the Ge films. For the growth temperature, the highest tensile strain is found to be in the range of $0.22-0.24 \%$. We have also shown that cyclic annealing is more efficient than hightemperature annealing and by controlling the annealing parameter, such as the number of annealing cycles and the annealing time, it is possible to obtain a tensile strain as high as $0.30 \%$. To our knowledge, this value represents the highest value ever reported in the $\mathrm{Ge} / \mathrm{Si}$ system. The photoluminescence intensity of the Ge direct band gap emission has been shown to be enhanced and a redshift of $\sim 19$ nmis observed due to a decrease of the Ge conduction band edge with the presence of tensile strain in the layers.

\section{ACKNOWLEDGMENTS}

This work has been carried out in the frame of a bilateral collaboration agreement signed between the Hong Duc University and the Aix-Marseille University (AMU). We thank Prof. V. Le Thanh at the AMU for his support to this work. 


\section{REFERENCES}

[1] J. Liu, X. Sun, R. Camacho-Aguilera, L. C. Kimerling, and J. Michel, Opt. Lett.35 (2010) 679 and references therein

[2] R. Soref, J. Kouvetakis, and J. Menendez, Mater. Res. Soc. Symp. Proc. 958 (2007) 13

[3] R. Soref, J. Kouvetakis, J. Tolle, J. Menendez, and V. D'Costa, J. Mater. Res. 22 (2007) 3281

[4] M. El Kurdi, G. Fishman, S. Sauvage, and P. Boucaud, J. Appl. Phys. 107 (2010)013710

[5] X. Sun, J.F. Liu, L.C. Kimerling, J. Michel, Appl. Phys. Lett. 95 (2009) 011911

[6] M. El Kurdi, T. Kociniewski, T.-P. Ngo, J. Boulmer, D. Débarre, P. Boucaud, J.F. Damlencourt, O. Kermarrec, and D. Bensahel, Appl. Phys. Lett. 94 (2009)191107

[7] M. El Kurdi, H. Bertin, E. Martincic, M. de Kersauson, G. Fishman, S. Sauvage, A. Bosseboeuf, and P. Boucaud, Appl. Phys. Lett.96 (2010)041909

[8] Y. Bai, K. E. Lee, C. Cheng, M. L. Lee, and E. A. Fitzgerald, J. Appl. Phys. 104 (2008) 084518

[9] Y.-Y. Fang, J. Tolle, R. Roucka, A.V.G. Chizmeshya, J. Kouvetakis, V.R. D'Costa, J. Menendez, Appl. Phys. Lett. 90, 061915 (2007); J. Mene“ndez, J. Kouvetakis, Appl. Phys. Lett. 85 (2004) 1175

[10] D.J. Eaglesham, M. Cerullo, Phys. Rev. Lett. 64 (1990) 1943

[11] V. Le Thanh, Surf. Sci. 492 (2001) 255 and references therein

[12] L. Colace, G. Masini, F. Galluzzi, G. Assanto, G. Capellini, L. Di Gaspare, E. Pelange, and F. Evangelisti, Appl. Phys. Lett.72 (1998) 3175

[13] H.-C. Luan, D.R. Lim, K.K. Lee, K.M. Chen, J.G. Sandland, K. Wada, L.C. Kimerling, Appl. Phys. Lett.75 (1999) 2009

[14] J.-M. Hartmann, A. Abbadie, A.M. Papon, P. Holliger, G. Rolland, T. Billon, J.M. Fedeli, M. Rouviere, L. Vivien, S. Laval, J. Appl. Phys.95 (2004)5905

[15] J.-M. Hartmann, A. M. Papon, V. Destefanis, T. Billon, J. Cryst. Growth. 310 (2008)5287

[16] V. Le Thanh, V. Aubry-Fortuna, D. Bouchier, A. Younsi, and G. Hincelin, Surf. Sci. 369 (1996) 85

[17] M. Halbwax, D. Bouchier, V. Yam, D. Debarre, Lam H. Nguyen, Y. Zheng, P. Rosner, M. Benamara, H. P. Strunk, C. Clerc, J. Appl. Phys. 97 (2005)064907

[18] V. Le Thanh, D. Bouchier, G. Hincelin, J. Appl. Phys. 87 (2000)3700

[19] J. Liu, D. D. Cannon, Y. Ishikawa, K. Wada, D. T. Danielson, S. Jongthammanurak, J. Michel, and L. C. Kimerling, Phys. Rev. B. 70 (2004) 155309

[20] J. Liu, H. J. Kim, O. Hul'ko, Y. H. Xie, S. Sahni, P. Bandaru, and E. Yablonovitch, J. Appl. Phys. 96 (2004) 916. 